\title{
Single-Stage Endoscopic Stone Extraction and Cholecystectomy during the Same Hospitalization
}

\author{
Toshiaki Terauchi ${ }^{1}$, Hiroharu Shinozaki ${ }^{1}$, Satoshi Shinozaki ${ }^{2,3}$, Yuichi Sasakura $^{1}$, Masaru Kimata $^{1}$, Junji Furukawa ${ }^{1}$, \\ Alan Kawarai Lefor ${ }^{4}$, Yoshiro Ogata' and Kenji Kobayashi ${ }^{1}$ \\ ${ }^{1}$ Department of Surgery, Saiseikai Utsunomiya Hospital, Tochigi, ${ }^{2}$ Shinozaki Medical Clinic, Tochigi, ${ }^{3}$ Division of Gastroenterology, \\ Department of Medicine, Jichi Medical University, Tochigi, ${ }^{4}$ Department of Surgery, Jichi Medical University, Tochigi, Japan
}

Background/Aims: The clinical impact of single-stage endoscopic stone extraction by endoscopic retrograde cholangiopancreatography (ERCP) and cholecystectomy during the same hospitalization remains elusive. This study aimed to determine the efficacy and safety of single-stage ERCP and cholecystectomy during the same hospitalization in patients with cholangitis.

Methods: We retrospectively reviewed the medical records of 166 patients who underwent ERCP for mild to moderate cholangitis due to choledocholithiasis secondary to cholecystolithiasis from 2012 to 2016.

Results: Complete stone extraction was accomplished in $92 \%$ of patients (152/166) at the first ERCP. Among 152 patients who underwent complete stone extraction, cholecystectomy was scheduled for 119 patients (78\%). Cholecystectomy was performed during the same hospitalization in $89 \%$ of patients (106/119). We compared two groups of patients: those who underwent cholecystectomy during the same hospitalization $(n=106)$ and those who underwent cholecystectomy during a subsequent hospitalization $(n=13)$. In the delayed group, cholecystectomy was performed about three months after the first ERCP. There were no significant differences between the groups in terms of operative time, rate of postoperative complications, and interval from cholecystectomy to discharge.

Conclusions: Single-stage endoscopic stone extraction is recommended in patients with mild to moderate acute cholangitis due to choledocholithiasis. The combination of endoscopic stone extraction and cholecystectomy during the same hospitalization is safe and feasible. Clin Endosc 2019;52:59-64

Key Words: Cholangitis; Choledocholithiasis; Cholangiopancreatography; Patient outcome assessment; Cholecystectomy, laparoscopic

\section{INTRODUCTION}

The updated Tokyo Guidelines 13 (TG13) standardize the diagnosis and treatment of patients with acute cholangitis associated with choledocholithiasis. ${ }^{1}$ Choledocholithiasis is the etiology in $48 \%$ of patients with acute cholangitis, and

Received: June 21, 2018 Revised: August 14, 2018

Accepted: August 27, 2018

Correspondence: Satoshi Shinozaki

Shinozaki Medical Clinic, 6-1-13 Kiyoharadai, Utsunomiya, Tochigi 321-3223, Japan

Tel: +81-28-667-7722, Fax: +81-28-667-7305, E-mail: shinozaki-s@aqua.ocn.ne.jp ORCID: https://orcid.org/0000-0001-6935-4470

cc This is an Open Access article distributed under the terms of the Creative Commons Attribution Non-Commercial License (http://creativecommons.org/ licenses/by-nc/3.0) which permits unrestricted non-commercial use, distribution, and reproduction in any medium, provided the original work is properly cited. it requires emergency endoscopic drainage. ${ }^{2}$ TG13 requires systemic inflammation, cholestasis, and imaging to establish the diagnosis of acute cholangitis. ${ }^{3}$ TG13 defines mild cholangitis as Grade I, moderate, as Grade II; and severe, as Grade III. ${ }^{3}$ Although TG13 states the timing and necessity of biliary drainage based on severity, it does not define the optimal number of therapeutic sessions of endoscopic retrograde cholangiopancreatography (ERCP) required to treat patients with acute cholangitis due to choledocholithiasis, or the optimal timing of cholecystectomy.

For treatment of patients with acute cholangitis due to choledocholithiasis secondary to cholecystolithiasis, single-stage ERCP with cholecystectomy during the same hospitalization seems preferable to reduce the physical and economic burdens on patients. Since 2000, we have attempted both single-stage endoscopic complete stone extraction and cholecystectomy 
during the same hospitalization at Saiseikai Utsunomiya Hospital. We try to perform ERCP within 24 hours after the diagnosis of acute cholangitis secondary to choledocholithiasis. The strength of this strategy is the requirement to perform a minimum number of ERCP procedures, thereby resulting in reduced medical expenditures; a weakness of this strategy is increased physical burden on the patient owing to the prolonged duration required to accomplish complete stone removal during the first $\mathrm{ERCP}$, compared with drainage alone. Eto et al. reported the clinical feasibility of single-stage endoscopic stone extraction. ${ }^{4}$ However, many institutions in Japan schedule multiple sessions of ERCP in patients with acute cholangitis due to choledocholithiasis and then plan cholecystectomy during a subsequent hospitalization. This study aimed to determine the efficacy and safety of single-stage ERCP in patients with mild to moderate acute cholangitis due to choledocholithiasis, and the feasibility of cholecystectomy during the same hospitalization.

\section{MATERIALS AND METHODS}

\section{Study population}

In our institution, 398 patients with acute cholangitis due to choledocholithiasis underwent ERCP with stone extraction from 2012 to 2016. Acute cholangitis was diagnosed based on the TG13 criteria. We reviewed the following data from medical records: medical history, laboratory findings, endoscopic findings, operation records, and clinical course. To obtain homogenous data, we applied the following exclusion criteria: Grade III acute cholangitis, history of intervention at the papilla, common bile duct debris without stones, complications of pancreatitis before ERCP, antiplatelet/anticoagulant therapy, impossibility to cannulate, placement of a biliary stent, cirrhosis, history of cholecystectomy, and pancreaticobiliary malformations. We excluded 232 patients, and the remaining 166 patients were included in the final cohort (Fig. 1). The primary endpoint of this study was the efficacy and safety of single-stage ERCP. The secondary endpoint was the feasibility of both ERCP and cholecystectomy during the same hospital stay. Our institutional review board approved this retrospective review (No. 2017-14).

\section{ERCP procedure}

We routinely plan complete stone extraction during a single ERCP session in order to reduce social and economic burdens on patients. We do not place an endoscopic nasobiliary drainage (ENBD) tube or endoscopic biliary stent as long as complete ductal clearance is confirmed by cholangiography during ERCP. However, we do not exclude ENBD tube place- ment if the endoscopist considers it necessary, as in the case of a massive purulent discharge from the bile duct or an obviously edematous papilla of Vater. If extraction of all stones was not attempted owing to poor general condition of the patient or in case of failed complete stone extraction during the first ERCP, an ENBD tube was inserted for biliary drainage, and ERCP was performed at a later date. Even if multiple sessions were expected during the first ERCP, we attempted to remove as many stones as possible at the first ERCP to reduce the burden at the second ERCP. Prior to ERCP, all patients underwent both abdominal ultrasound and computed tomography imaging. ERCP was performed under conscious sedation, using midazolam and pethidine. After successful cannulation, endoscopic sphincterotomy or endoscopic papillary balloon dilation were performed to facilitate stone removal. We routinely used a wire-guided mechanical lithotripter to avoid incarceration in the bile duct, and the stones were occasionally crushed.

\section{Cholecystectomy}

The indication for cholecystectomy after choledocholithiasis is the presence of stones secondary to cholecystolithiasis. Residual gallbladder stones or debris, or findings of cholecystitis on abdominal ultrasound and/or endoscopic ultrasonography is consistent with secondary stones. Laparoscopic cholecystectomy is the first choice of surgical approach. An elective laparotomy with biliary tract intervention was considered in patients with severe bile duct dilatation due to prior laparotomy. Conversion to laparotomy during a laparoscopic procedure was considered when severe peritoneal adhesions or unexpected bleeding was present.

\section{Statistical analysis}

BellCurve for Excel 2015 software (Social Survey Research Information Co. Ltd., Tokyo, Japan) was used for analyses. Categorical data were assessed using the chi-square test. When the expected frequency was less than five, Fisher's exact test was used. Data with a normal distribution were compared using Student's $t$-test, and data without a normal distribution were compared using Mann-Whitney's $U$-test. Differences between variables with $p<0.05$ were considered significant.

\section{RESULTS}

\section{Complete stone extraction at first ERCP}

The baseline characteristics and outcomes of the 166 study patients are shown in Table 1. We evaluated the clinical impact of single-stage ERCP for patients with mild to moderate acute cholangitis due to choledocholithiasis secondary to cho- 


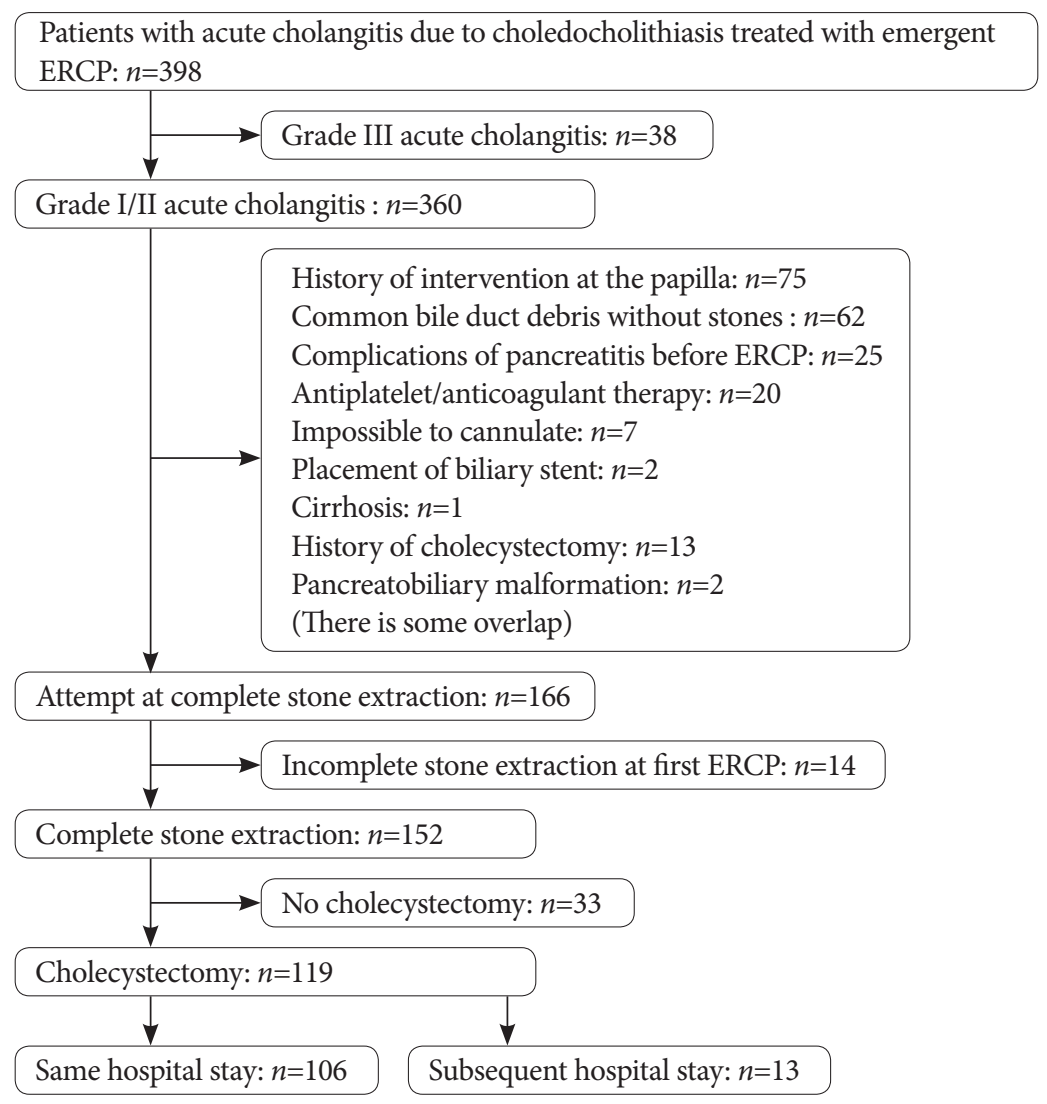

Fig. 1. Study flowchart. ERCP, endoscopic retrograde cholangiopancreatography.

lecystolithiasis. All stones were successfully extracted in 152 of 166 patients (92\%) (Fig. 1). The reasons for incomplete stone removal included large stone size $(n=7)$, long duration of the procedure $(n=6)$, and massive purulent discharge during the ERCP $(n=1)$. As long as all stones were extracted, an ENBD tube was not inserted. Of the 152 patients who underwent successful stone extraction, 119 (78\%) underwent cholecystectomy, and the remaining 33 patients (22\%) did not undergo surgery owing to advanced age and/or severe background disease.

\section{Cholecystectomy and postoperative course}

Of the 119 patients who underwent cholecystectomy, we compared patients who underwent cholecystectomy during the same hospitalization $(n=106)$ with those who underwent cholecystectomy during a subsequent hospitalization $(n=13)$ (Fig. 1). The reasons for undergoing cholecystectomy during a subsequent hospitalization were as follows: patient desire $(n=9)$, preoperative cardiovascular intervention $(n=2)$, preoperative anticoagulant therapy for venous thrombosis $(n=1)$, and the presence of a gastric ulcer $(n=1)$. Baseline characteristics and ERCP procedures were similar in the two groups (Table 2). The interval from ERCP to operation in patients who underwent cholecystectomy during the same hospitalization was significantly shorter than the corresponding interval in patients who underwent cholecystectomy during a subsequent hospitalization after three months $(p<0.001)$. The operative time, incidence of postoperative complications, and interval from operation to discharge did not differ in the two groups.

\section{DISCUSSION}

This retrospective cohort study shows that single-stage stone extraction by ERCP in patients with mild to moderate cholangitis due to choledocholithiasis is safe and feasible, and it shows a satisfactory rate of complete stone removal (92\%). Cholecystectomy during the same hospitalization is accomplished safely in most patients. This strategy can shorten the time interval from ERCP to operation, and it may reduce the recurrence of choledocholithiasis in the period from ERCP to operation. We believe that single-stage stone extraction by ERCP and cholecystectomy during the same hospitalization are feasible and can be the recommended strategy.

Few studies have reported on the feasibility of emergen- 
cy single-stage endoscopic treatment for cholangitis due to choledocholithiasis. Undoubtedly, biliary decompression should be performed as early as possible, because delayed drainage by ERCP increases the likelihood of organ failure and emergency readmission. ${ }^{5,6}$ In patients with severe cholangitis such as Grade III cholangitis (as defined in TG13), we do not attempt single-stage ERCP. We perform only biliary drainage to complete the ERCP procedure as soon as possible without complete stone extraction in such patients. Further, the primary endpoint of this study was the efficacy and safety of single-stage ERCP for patients with choledocholithiasis. Therefore, patients with Grade III cholangitis were not included in this study. Eto et al. reported the feasibility and safety of single-stage endoscopic treatment for non-severe cholangitis

Table 1. Patient Characteristics

\begin{tabular}{|c|c|}
\hline \multicolumn{2}{|l|}{ Baseline characteristics before ERCP } \\
\hline Age, yr, mean \pm SD & $69 \pm 13$ \\
\hline Gender, male, $n$ & $104(63 \%)$ \\
\hline Grade of acute cholangitis, grade $1 / 2, n$ & $82(49 \%) / 84(51 \%)$ \\
\hline Number of stones & $2.1 \pm 1.4$ \\
\hline Maximum diameter of stone, $\mathrm{mm}$ & $6.0 \pm 4.1$ \\
\hline $\begin{array}{l}\text { Comorbidities, } n \\
\text { Hypertension } \\
\text { Diabetes mellitus } \\
\text { Cardiovascular disease }\end{array}$ & $\begin{array}{l}75(45 \%) \\
20(12 \%) \\
39(23 \%)\end{array}$ \\
\hline Body temperature, $\geq 38^{\circ} \mathrm{C}, n$ & $42(25 \%)$ \\
\hline White blood cell count, $\times 10^{3} / \mu \mathrm{L}$ & $11.8 \pm 4.1$ \\
\hline Hemoglobin, g/dL & $13.4 \pm 1.6$ \\
\hline Platelet count, $\times 10^{4} / \mu$ & $21.9 \pm 8.5$ \\
\hline Total bilirubin, mg/dL & $3.5 \pm 2.3$ \\
\hline Direct bilirubin, mg/dL & $2.4 \pm 1.9$ \\
\hline Aspartate aminotransferase, U/L & $221 \pm 215$ \\
\hline Alanine aminotransferase, $\mathrm{U} / \mathrm{L}$ & $241 \pm 191$ \\
\hline Alkaline phosphatase, U/L & $786 \pm 581$ \\
\hline$\gamma$-glutamyl transferase, $\mathrm{U} / \mathrm{L}$ & $547 \pm 454$ \\
\hline ERCP procedure & \\
\hline Total procedure time required for ERCP, min & $18.4 \pm 12.3$ \\
\hline ERCP time except cannulation time, min & $15.2 \pm 10.3$ \\
\hline $\begin{array}{l}\text { Intervention to papilla, } n \\
\text { Balloon dilation } \\
\text { Endoscopic sphincterotomy }\end{array}$ & $\begin{array}{l}62(37 \%) \\
96(55 \%)\end{array}$ \\
\hline Complete stone removal, $n$ & $152(92 \%)$ \\
\hline $\begin{array}{l}\text { Adverse events, } n \\
\text { Pancreatitis }\end{array}$ & $5(3 \%)$ \\
\hline
\end{tabular}

ERCP, endoscopic retrograde cholangiopancreatography; SD, standard deviation. due to choledocholithiasis based on a prospective study. ${ }^{4}$ Saito et al. reported the feasibility and safety of single-stage endoscopic stone extraction in patients with choledocholithiasis. ${ }^{7}$ Single-stage treatment was not a significant risk factor for ERCP-related complications, and it significantly shortened the hospital stay. ${ }^{7}$ Although the present study is retrospective, the number of patients who underwent cholecystectomy during the same hospitalization is large. We recommend single-stage endoscopic stone extraction as the standard strategy for treatment of patients with non-severe cholangitis due to choledocholithiasis.

In Japan, endoscopic drainage and stone removal are generally performed separately over multiple endoscopic sessions. The Japanese health insurance system reimburses only drainage or stone removal during one session, and both are not reimbursed simultaneously. Multiple sessions for ERCP and cholecystectomy during a subsequent hospitalization are performed only to incur profits for the medical institution. Multiple-stage ERCP and cholecystectomy performed during a subsequent hospitalization cost approximately 600,000 Japanese Yen (about \$5,400 US) more than the cost incurred when single-stage ERCP and cholecystectomy are performed during the same hospitalization. Thus, performing single-stage ERCP and cholecystectomy during the same hospitalization not only minimizes the social burden on the patient but also decreases costs.

We usually perform biliary cannulation using a pull-type sphincterotome, which can control the angle for cannulation as well as enable a sphincterotomy. ${ }^{8}$ Endoscopic sphincterotomy provides spontaneous duct drainage and allows small stones to pass spontaneously. Hui et al. reported that a plastic stent is not necessary after endoscopic sphincterotomy in patients with acute cholangitis due to choledocholithiasis, in a randomized controlled trial. ${ }^{9}$ Endoscopic sphincterotomy has a higher success rate for complete stone clearance and less requirement for mechanical lithotripsy than endoscopic papillary balloon dilation does. ${ }^{8,10}$ We recommend the use of endoscopic sphincterotomy during single-stage ERCP for patients with choledocholithiasis.

There are various considerations regarding the treatment algorithm for patients with cholangitis due to choledocholithiasis from ERCP to cholecystectomy. A recent study from the United States showed the safety and economic superiority of ERCP and laparoscopic cholecystectomy performed during the same session under general anesthesia, and a study from China also showed similar results. ${ }^{11,12}$ The British Society of Gastroenterology guidelines for the management of common bile duct stones suggest laparoscopic stone removal and cholecystectomy. ${ }^{13}$ However, laparoscopic treatment for choledocholithiasis requires a long time, special training, and 
Table 2. Patient Characteristics and Outcomes

\begin{tabular}{|c|c|c|c|}
\hline & $\begin{array}{c}\text { Same hospital stay } \\
(n=106)\end{array}$ & $\begin{array}{l}\text { Another hospital stay } \\
\qquad(n=13)\end{array}$ & $p$-value \\
\hline \multicolumn{4}{|l|}{ Baseline characteristics before ERCP } \\
\hline Age, yr, mean \pm SD & $68 \pm 13$ & $73 \pm 9$ & 0.068 \\
\hline Gender, male, $n$ & $66(62 \%)$ & $8(62 \%)$ & 0.959 \\
\hline Grade of acute cholangitis, grade $1 / 2, n$ & $60 / 46$ & $4 / 9$ & 0.078 \\
\hline Number of stones & $1.9 \pm 1.1$ & $2.5 \pm 1.4$ & 0.091 \\
\hline Maximum diameter of stones, $\mathrm{mm}$ & $5.4 \pm 3.4$ & $5.0 \pm 3.8$ & 0.707 \\
\hline $\begin{array}{l}\text { Comorbidities, } n \\
\text { Hypertension } \\
\text { Diabetes mellitus } \\
\text { Cardiovascular disease }\end{array}$ & $\begin{array}{l}50(47 \%) \\
14(13 \%) \\
15(14 \%)\end{array}$ & $\begin{array}{l}5(38 \%) \\
2(15 \%) \\
4(31 \%)\end{array}$ & $\begin{array}{l}0.552 \\
0.828 \\
0.123\end{array}$ \\
\hline Body temperature, $>38^{\circ} \mathrm{C}, n$ & $18(17 \%)$ & $4(31 \%)$ & 0.313 \\
\hline White blood cell count, $\times 10^{3} / \mu \mathrm{L}$ & $11.8 \pm 3.9$ & $13.4 \pm 3.8$ & 0.149 \\
\hline Hemoglobin, g/dL & $13.5 \pm 1.6$ & $14.0 \pm 1.6$ & 0.370 \\
\hline Platelet count, $\times 10^{4} / \mu$ & $21.7 \pm 7.7$ & $19.9 \pm 6.0$ & 0.425 \\
\hline Total bilirubin, mg/dL & $3.5 \pm 2.6$ & $4.1 \pm 1.9$ & 0.404 \\
\hline Direct bilirubin, mg/dL & $2.3 \pm 2.0$ & $3.1 \pm 1.7$ & 0.196 \\
\hline Aspartate aminotransferase, U/L & $213 \pm 202$ & $227 \pm 266$ & 0.824 \\
\hline Alanine aminotransferase, U/L & $241 \pm 187$ & $217 \pm 219$ & 0.662 \\
\hline Alkaline phosphatase, U/L & $738 \pm 591$ & $987 \pm 540$ & 0.154 \\
\hline$\gamma$-glutamyl transferase, $\mathrm{U} / \mathrm{L}$ & $545 \pm 447$ & $637 \pm 554$ & 0.498 \\
\hline \multicolumn{4}{|l|}{ ERCP procedure } \\
\hline Total procedure time of ERCP, min & $17.1 \pm 10.7$ & $19.4 \pm 15.3$ & 0.491 \\
\hline ERCP time except cannulation time, min & $14.3 \pm 9.0$ & $17.5 \pm 13.3$ & 0.266 \\
\hline $\begin{array}{l}\text { Intervention to papilla, } n \\
\text { Balloon dilation } \\
\text { Sphincterotomy }\end{array}$ & $\begin{array}{l}44(42 \%) \\
62(58 \%)\end{array}$ & $\begin{array}{l}6(46 \%) \\
7(54 \%)\end{array}$ & 0.749 \\
\hline $\begin{array}{l}\text { Adverse events, } n \\
\text { Pancreatitis }\end{array}$ & $3(3 \%)$ & $0(0 \%)$ & $1.000^{\mathrm{a})}$ \\
\hline \multicolumn{4}{|l|}{ After ERCP } \\
\hline Time for normalization of body temperature, days, median (IQR) & $\begin{array}{l}1(1-2) \\
(n=18)\end{array}$ & $\begin{array}{c}2(1-3) \\
(n=4)\end{array}$ & $0.451^{\mathrm{b})}$ \\
\hline Interval from ERCP to discharge, days, median (IQR) & - & $8(6-10)$ & \\
\hline Interval from ERCP to operation, days, median (IQR) & $6(4-7)$ & $98(62-206)$ & $<0.001^{\text {b) }}$ \\
\hline \multicolumn{4}{|l|}{ Cholecystectomy } \\
\hline $\begin{array}{l}\text { Operative time, min } \\
\text { Laparoscopic cholecystectomy, } n \\
\text { Intraoperative conversion to laparotomy, } n \\
\text { Elective laparotomy, } n \\
\text { Postoperative complications, } n \\
\text { Interval from operation to discharge, days, median (IQR) }\end{array}$ & $\begin{array}{c}99 \pm 42 \\
100(94 \%) \\
2(2 \%) \\
4(4 \%) \\
0 \\
3(3-5)\end{array}$ & $\begin{array}{c}104 \pm 33 \\
13(100 \%) \\
0 \\
0 \\
1(8 \%)^{c)} \\
3(3-4)\end{array}$ & $\begin{array}{l}0.676 \\
0.620^{\mathrm{a})} \\
1.000^{\mathrm{a}} \\
1.000^{\mathrm{a}} \\
0.109^{\mathrm{a}} \\
0.898^{\mathrm{b}}\end{array}$ \\
\hline
\end{tabular}

ERCP, endoscopic retrograde cholangiopancreatography; SD, standard deviation; IQR, interquartile range.

${ }^{\text {a) }}$ Fisher's exact test.

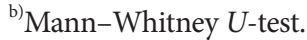

${ }^{c)}$ Intraperitoneal abscess. 
expensive dedicated devices. ${ }^{14}$ Conscious sedation during ERCP is commonly practiced in Japan, and Japanese guidelines recommend conscious sedation for routine ERCP, except in cases where a stable level of sedation for a long duration is required. ${ }^{15}$ As a universal strategy, single-stage endoscopic treatment with cholecystectomy may be preferred worldwide. The present study shows that ERCP under conscious sedation and cholecystectomy under general anesthesia during the same hospitalization are safe and feasible.

This study has some limitations that should be acknowledged. First, this is a single-center retrospective study, and the number of study subjects in the interval cholecystectomy group may be too small to draw a definitive conclusion. Further large-scale studies are necessary to confirm these preliminary results. Second, the decisions regarding discharge from the hospital, and cholecystectomy were made by the physician in charge. Third, patients undergoing cholecystectomy during a subsequent hospitalization had their own reasons for not undergoing the procedure during the same hospitalization. However, an interventional study, such as a randomized controlled study, is not feasible to investigate this issue. The benefit of cholecystectomy during the same hospitalization is obvious, and a long interval between complete stone extraction and cholecystectomy increases the likelihood of recurrent choledocholithiasis.

In conclusion, single-stage endoscopic complete stone extraction is recommended for patients with mild to moderate acute cholangitis due to choledocholithiasis. Performance of cholecystectomy during the same hospitalization does not affect the operative time, incidence of postoperative complications, or interval between the cholecystectomy and discharge. Endoscopic stone extraction and cholecystectomy during the same hospitalization are feasible, and they can help reduce the physical and economic burden on patients.

\section{Conflicts of Interest}

The authors have no financial conflicts of interest.

\section{REFERENCES}

1. Miura F, Takada T, Strasberg SM, et al. TG13 flowchart for the management of acute cholangitis and cholecystitis. J Hepatobiliary Pancreat Sci 2013;20:47-54.

2. Gigot JF, Leese T, Dereme T, Coutinho J, Castaing D, Bismuth H. Acute cholangitis. Multivariate analysis of risk factors. Ann Surg 1989;209:435438 .

3. Kiriyama S, Takada T, Strasberg SM, et al. TG13 guidelines for diagnosis and severity grading of acute cholangitis (with videos). J Hepatobiliary Pancreat Sci 2013;20:24-34.

4. Eto K, Kawakami H, Haba S, et al. Single-stage endoscopic treatment for mild to moderate acute cholangitis associated with choledocholithiasis: a multicenter, non-randomized, open-label and exploratory clinical trial. J Hepatobiliary Pancreat Sci 2015;22:825-830.

5. Khashab MA, Tariq A, Tariq U, et al. Delayed and unsuccessful endoscopic retrograde cholangiopancreatography are associated with worse outcomes in patients with acute cholangitis. Clin Gastroenterol Hepatol 2012;10:1157-1161.

6. Navaneethan U, Gutierrez NG, Jegadeesan R, et al. Delay in performing ERCP and adverse events increase the 30-day readmission risk in patients with acute cholangitis. Gastrointest Endosc 2013;78:81-90.

7. Saito H, Kadono Y, Kamikawa K, et al. The incidence of complications in single-stage endoscopic stone removal for patients with common bile duct stones: a propensity score analysis. Intern Med 2018;57:469-477.

8. Ramchandani M, Pal P, Reddy DN. Endoscopic management of acute cholangitis as a result of common bile duct stones. Dig Endosc 2017;29 Suppl 2:78-87.

9. Hui CK, Lai KC, Yuen MF, et al. Does the addition of endoscopic sphincterotomy to stent insertion improve drainage of the bile duct in acute suppurative cholangitis? Gastrointest Endosc 2003;58:500-504.

10. Weinberg BM, Shindy W, Lo S. Endoscopic balloon sphincter dilation (sphincteroplasty) versus sphincterotomy for common bile duct stones. Cochrane Database Syst Rev 2006;(4):CD004890.

11. Mallick R, Rank K, Ronstrom C, et al. Single-session laparoscopic cholecystectomy and ERCP: a valid option for the management of choledocholithiasis. Gastrointest Endosc 2016;84:639-645.

12. Zang JF, Zhang C, Gao JY. Endoscopic retrograde cholangiopancreatography and laparoscopic cholecystectomy during the same session: feasibility and safety. World J Gastroenterol 2013;19:6093-6097.

13. Williams E, Beckingham I, El Sayed G, et al. Updated guideline on the management of common bile duct stones (CBDS). Gut 2017;66:765-782.

14. Costi R, Gnocchi A, Di Mario F, Sarli L. Diagnosis and management of choledocholithiasis in the golden age of imaging, endoscopy and laparoscopy. World J Gastroenterol 2014;20:13382-13401.

15. Obara K, Haruma K, Irisawa A, et al. Guidelines for sedation in gastroenterological endoscopy. Dig Endosc 2015;27:435-449. 